\title{
Corynebacterium genitalium
}

National Cancer Institute

\section{Source}

National Cancer Institute. Corynebacterium genitalium. NCI Thesaurus. Code C86313.

A species of aerobic or facultatively anaerobic, Gram-positive, coryneform, pleomorphic bacilli assigned to the phylum Actinobacteria. This species is nonmotile, non-spore forming, catalase positive, and not acid fast. Some C. genitalium strains are commensal in the urinary tract while other strains are associated with urinary tract infections. 